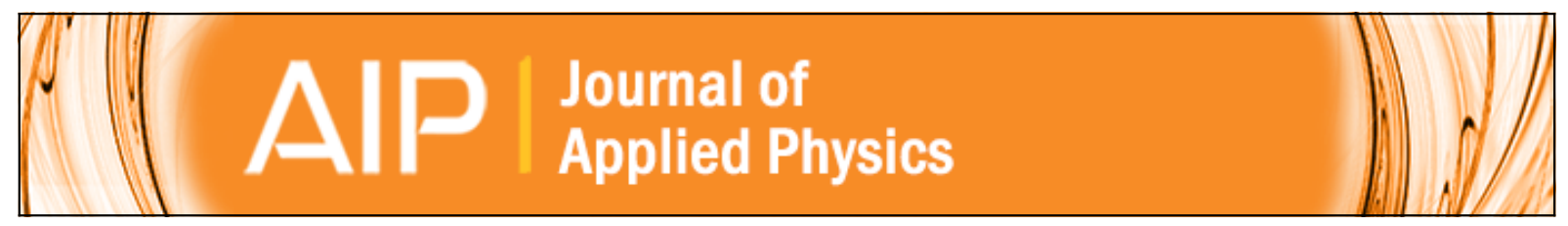

\title{
Size effects in the magnetization of a superconducting wire
}

Pablo A. Venegas

Citation: Journal of Applied Physics 85, 6049 (1999); doi: 10.1063/1.369078

View online: http://dx.doi.org/10.1063/1.369078

View Table of Contents: http://scitation.aip.org/content/aip/journal/jap/85/8?ver=pdfcov

Published by the AIP Publishing

\section{Articles you may be interested in}

Size and polydispersity effect on the magnetization of densely packed magnetic nanoparticles

J. Appl. Phys. 112, 073926 (2012); 10.1063/1.4757418

Flux-cutting and flux-transport effects in type-Il superconductor slabs in a parallel rotating magnetic field Low Temp. Phys. 37, 947 (2011); 10.1063/1.3672157

Fundamental microwave-power-limiting mechanism of epitaxial high-temperature superconducting thin-film devices

J. Appl. Phys. 97, 113911 (2005); 10.1063/1.1929088

Critical currents, phase slip centers, and phase slip lines in superconducting films in the absence of external magnetic field

Low Temp. Phys. 31, 127 (2005); 10.1063/1.1820552

Surface impedance of a thin superconducting film in a parallel magnetic field Low Temp. Phys. 27, 333 (2001); 10.1063/1.1374716

\section{AlP Re-register for Table of Content Alerts}

\section{Create a profile.

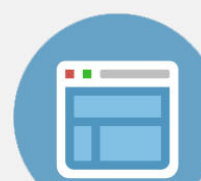 \\ Sign up today!}




\title{
Size effects in the magnetization of a superconducting wire
}

\author{
Pablo A. Venegas ${ }^{\text {a) }}$ \\ Physics Department, University of California, Santa Cruz, California 95064
}

The size effects in the magnetization of a long cylindrical wire of circular cross section in the presence of an external magnetic field are investigated. For this study the London theory is used with boundary conditions appropriate for this geometry. Using the Monte Carlo simulated annealing method, the free energy of the mixed state is minimized with respect to the vortex positions. The ground state of the vortex lattice for $n=1$ up to 18 vortices for a given radius of the cylinder is obtained. It is found that the finite size of the sample provokes a matching effect in the magnetization, as found in experiments with superconducting samples of finite size but different geometry. (c) 1999 American Institute of Physics. [S0021-8979(99)16408-9]

Over the last few years several studies on superconducting samples of size comparable with the London penetration depth have been made. Measures of transport currents, magnetization, magnetic moment, reversibility lines, and flux penetration ${ }^{1-12}$ in samples of small size have shown the importance of finite size effects. As an example the results of Zeldov et $a .^{7}$ can be cited in thin superconducting strips that show a delay in the penetration of the vortex lines and a vortex accumulation at the center of the sample due to the geometrical barrier effect. On the other hand, studies of magnetization made by Brongersma et al. ${ }^{12}$ and Ziese et al. ${ }^{13}$ in superconducting films show an interesting behavior. When the magnetic field is increased above the lower critical field $\left(H_{c 1}\right)$, instead of the continuous decrease in bulk superconductors, it is found that magnetization shows a series of maxima (matching effect) which is attributed to finite size effects. In this way, our main interest in this work is to numerically analyze the matching effects in the magnetization. Instead of a film, these effects are analyzed in an infinite superconducting wire of circular cross section. Despite the different geometry a matching effect analog is found in thin films.

Suppose we have an infinite cylindrical superconductor of radius $a$ along the $z$ axis in an external magnetic field oriented along the $z$ direction. If we suppose we are in the high $\kappa$ regime then the London theory can be used to analyze the present system (where $\kappa$ is the Ginzburg-Landau parameter $\kappa=\lambda / \xi$, $\xi$ is the coherence length, and $\lambda$ the penetration length). To calculate the magnetization it is necessary to know the vortex lattice associated to the ground state of the system, which is obtained by minimizing the Gibbs free energy with respect to the vortex positions using the Monte Carlo simulated annealing method. The ground state lattice patterns are obtained starting from an arbitrary configuration allowing the system to evolve towards the global minimum.

If we suppose our cylinder is isotropic then the vortices are straight lines and the three dimensional problem can be reduced to a two dimensional one. Writing the London equation in cylindrical polar coordinates $(r, \phi)$ with boundary

\footnotetext{
a) On leave from Departamento de Fisica, Universidade Estadual Paulista,
} Av. Engenheiro Luiz E. Coube S/N, 17033-360 Bauru-SP, Brazil. conditions appropriated to this geometry, the Gibbs free energy for the system of $N$ vortices in an external field $H$ can be written as, ${ }^{13}$

$$
\begin{aligned}
\mathcal{G}= & \left(\frac{\Phi_{0}}{4 \pi \lambda^{2}}\right)^{2} \frac{\lambda^{2}}{A}\left[N \ln \kappa+\sum_{i \neq j} K_{0}\left(\left|\mathbf{r}_{i}-\mathbf{r}_{j}\right| / \lambda\right)\right. \\
& -\sum_{i, j} \sigma\left(r_{i}, \phi_{i}, r_{j}, \phi_{j}\right)+\widetilde{H} \sum_{i}\left(\frac{I_{0}\left(r_{i} / \lambda\right)}{I_{0}(a / \lambda)}-1\right) \\
& \left.-\left(\frac{\tilde{H}}{2}\right)^{2} \frac{a}{\lambda} \frac{I_{1}(a / \lambda)}{I_{0}(a / \lambda)}\right] .
\end{aligned}
$$

The associated induction is then given by

$$
\begin{aligned}
B= & \frac{N \Phi_{0}}{A}+\frac{2 \pi a \lambda H}{A} \frac{I_{1}(a / \lambda)}{I_{0}(a / \lambda)} \\
& -\frac{\Phi_{0}}{A} \frac{1}{I_{0}(a / \lambda)} \sum_{i} I_{0}\left(r_{i} / \lambda\right),
\end{aligned}
$$

where the definition used was

$$
\begin{aligned}
& \sigma\left(r, \phi, r^{\prime}, \phi^{\prime}\right) \\
& \quad=\sum_{m=-\infty}^{+\infty} \cos \left[m\left(\phi-\phi^{\prime}\right)\right] \frac{K_{m}(a / \lambda)}{I_{m}(a / \lambda)} I_{m}(r / \lambda) I_{m}\left(r^{\prime} / \lambda\right),
\end{aligned}
$$

$I_{m}$ and $K_{m}$ are the modified Bessel functions, $r_{i}$ the vortex position, $A$ the area of the cylinder, and $\tilde{H}=H /\left(\Phi_{0} / 4 \pi \lambda^{2}\right)$.

The result obtained for the Gibbs free energy reflects the different interactions in our finite size system. The first term is the vortex self-energy and the second one describes the repulsive interaction between the vortices (the bulk term). The third term describes the attractive interaction between the vortices and image vortices located outside the sample. The effect of this interaction is to push the vortices close to the surface. The argument of the fourth term represents the effective flux $\Phi / \Phi_{0}=\left[1-I_{0}(r) / I_{0}(a)\right]\left(\Phi_{0}=h c / 2 e\right)$. The first term in this argument represents the repulsive interaction between a vortex and the magnetic field that penetrates the sample surface pushing the vortices to its center. The second term represents the vortex magnetic energy. The fifth term is the Meissner state energy. The competition between 
the vortex-vortex, vortex-image vortex, and vortex-surface field interactions represents an energy barrier that the vortex must overcome to be able to enter the sample. When the external field is below the minimum value for a new vortex penetration, the vortex-image vortex interaction dominates preventing a new vortex to enter the sample.

In this way, as a consequence of the energy barrier generated by the finite size of the sample, at low fields (near $H_{c 1}$ ), we have well defined critical fields for each new penetration, known as matching fields $\left[\tilde{H}_{s N}=H_{s N} /\left(\Phi_{0} / 4 \pi \lambda^{2}\right)\right.$, $N=1, \ldots, 18$; with $\left.\widetilde{H}_{s 1} \equiv \tilde{H}_{c 1}\right]$. When the field is increased up to or above the critical value a new vortex can overcome the surface energy barrier and enters into the sample. Then the first step to calculate the magnetization is to determine the matching fields.

The lowest matching field $H_{c 1}$ can be obtained easily comparing the free energy of the system with one vortex and that of the Meissner state (suppose the single vortex is at the center of the sample). The Meissner energy is obtained by solving the London equation in cylindrical coordinates of the system with no vortices. The lower critical field is then given by

$$
H_{c 1}=\frac{\Phi_{0}}{4 \pi \lambda^{2}}\left[\frac{\ln \kappa-\frac{K_{0}(a / \lambda)}{I_{0}(a / \lambda)}}{1-\frac{1}{I_{0}(a / \lambda)}}\right] .
$$

To obtain the matching fields for higher vortex configurations $(N>1)$ is more cumbersome but the procedure is essentially the same as used to obtain $\widetilde{H}_{c 1}$, i.e., equating the Gibbs free energy of the configurations with $N$ and $N+1$ vortices, $G_{N}=G_{N+1}$. In this way a transcendental equation is obtained for $\tilde{H}$ (because the radii implicitly depend on $\tilde{H}$ ) which can be solved iteratively. However, each time the free energy is calculated for a given magnetic field, this free energy must be calculated with the vortices in the equilibrium position. Thus, for each iteration the free energy must be minimized. The iterative work was performed using the secant method and the minimization using Monte Carlo simulated annealing. ${ }^{14,15}$ For $a / \lambda=10$ the values obtained for $\tilde{H}_{s N}$ can be seen in Table I.

The usual procedure to find the ground state of the vortex lattice is to assume some particular geometry and then evaluate the Gibbs free energy. The configuration corresponding to the lowest energy value is assumed as the most stable vortex lattice. Other authors ${ }^{11,16}$ have used the method of images to determine the vortex configuration. Using the Monte Carlo simulated annealing method, an initial configuration is started and chosen randomly and we allow the vortex lattice to evolve towards the global minimum. The energy minimization is made using different initial configurations and different seeds for the random number generator. In this way different annealing schedules are obtained which assure that the system goes to the global minimum.

Note that due to the symmetry of the problem, the free energy is invariant under any rotation, therefore one vortex
TABLE I. The first column is the number of vortices $(N)$ and the second column represents the values of the critical fields for each configuration of $N$ vortices. The critical fields were determined with a precision of $10^{-7}$.

\begin{tabular}{rc}
\hline \hline$N$ & $H_{s N}$ \\
\hline 1 & 3.6903209 \\
2 & 3.7037780 \\
3 & 3.7047593 \\
4 & 3.7157228 \\
5 & 3.7313060 \\
6 & 3.7468671 \\
7 & 3.7489583 \\
8 & 3.7722957 \\
9 & 3.8003082 \\
10 & 3.8088703 \\
11 & 3.8258413 \\
12 & 3.8335742 \\
13 & 3.8639324 \\
14 & 3.8702223 \\
16 & 3.9075828 \\
17 & 3.9106818 \\
18 & 3.9419102 \\
\hline
\end{tabular}

can be fixed along the $x$ axis so that the minimization procedure only involves $2 N-1$ variables. This has been done for $N=1$ up to $N=18$.

Then, having the critical fields (see Table I) the free energy is minimized in the appropriate range of field. The minimization provides the most stable vortex configuration to obtain the induction and then the magnetization. The magnetization can be written as

$$
-4 \pi M=B-H,
$$

and the result can be seen in Fig. 1. From Fig. 1 we can see that the magnetization shows a quite different behavior when compared with the bulk case. Instead of a continuous decrease when the magnetic field is increased on our finite size sample the magnetization decreases by steps. Even though the cylindrical symmetry and a parallel field is specialized it seems that there is an analogy between the Brongersma ${ }^{12}$ experimental result for the magnetization and the present one. According to our results, the series of "maxima" they

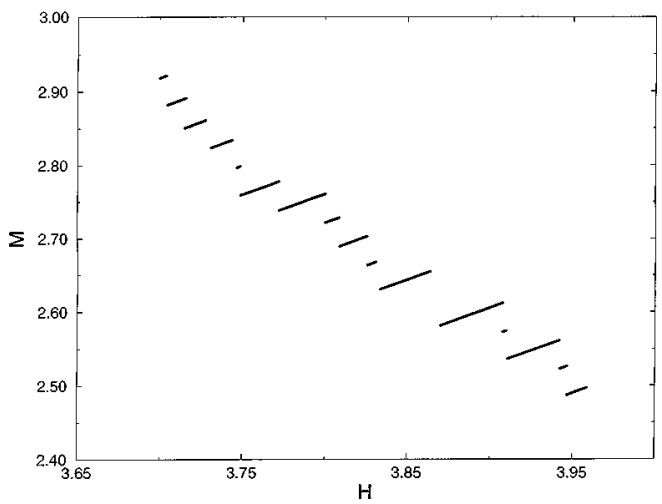

FIG. 1. Magnetization $(M)$ for $N=1-18$ vortices as a function of the external magnetic field $(H)$ in units of $\left(\Phi_{0} / 4 \pi \lambda^{2}\right)$. For each $N$ vortice configuration, the magnetization is calculated in the $\left[H_{s N}, H_{s N+1}\right]$ field range. Here we have used $a / \lambda=10, \lambda=200 \mathrm{~nm}$, and $\kappa=40$. 
found in their experiments can be interpreted as a function of the "jumps" shown in our calculations. The magnetization decreases as a function of $H$ when we go from one vortex configuration to a higher one, but the magnetization of each individual configuration of vortices increases with $H$, showing a maximum close to the next (higher) critical field. This series of maxima are analogous to those found in Brongersma experiments, suggesting that this is a consequence of the finite size of the samples and not an artifact of the used geometry.

The author would like to thank the Brazilian Agency Capes for partial financial support (Project No. 0750/96-4).

${ }^{1}$ See, for example, E. Guyon, in Superconductivily, edited by P. R. Wallace (Gordon and Breach, New York, 1969), and references therein.

${ }^{2}$ E. H. Brandt, Rep. Prog. Phys. 58, 1465 (1995), and references therein.

${ }^{3}$ E. H. Brandt, Phys. Rev. B 46, 8628 (1992).

${ }^{4}$ E. H. Brandt and M. Indembom, Phys. Rev. B 48, 12893 (1993).

${ }^{5}$ T. Schuster, H. Kuhn, E. H. Brandt, and S. Klaumünzer, Phys. Rev.
B 56, 3413 (1997).

${ }^{6}$ E. Zeldov, J. R. Clem, M. McElfresh, and M. Darvin, Phys. Rev. B 49, 9802 (1994).

${ }^{7}$ E. Zeldov, A. I. Larkin, V. B. Geshkenbein, M. Konczykowsky, D. Majer, B. Khaykovich, V. M. Vinokur, and H. Shtrikman, Phys. Rev. Lett. 73, 1428 (1994).

${ }^{8}$ B. P. Thrane, C. Schlenker, J. Dumas, and R. Buder, Phys. Rev. B 54, 15518 (1996).

${ }^{9}$ L. Civale, T. K. Worthington, and A. Gupta, Phys. Rev. B 43, 5425 (1991).

${ }^{10}$ B. Kaykovich, E. Zeldon, M. Konczykowsky, D. Majer, A. I. Larkin, and J. R. Clem, Physica C 235-240, 2757 (1994).

${ }^{11}$ S. H. Brongersma, E. Verweij, N. J. Koeman, D. G. de Groot, R. Griessen, and B. I. Ivlev, Phys. Rev. Lett. 71, 2319 (1993).

${ }^{12}$ M. Ziese, P. Esquinazi, P. Wangner, H. Adrian, S. H. Brongersma, and G. Griessen, Phys. Rev. B 53, 8658 (1996).

${ }^{13}$ P. A. Venegas and E. Sardella, Phys. Rev. B 58, 5789 (1998).

${ }^{14}$ W. H. Press, B. P. Flannery, S. A. Teukolsky, and W. T. Vetterling, Numerical Recipes (Cambridge University Press, Cambridge, 1992).

${ }^{15}$ S. Kirkpatrick, C. D. Gelatt, Jr., and M. P. Vecchi, Science 220, 671 (1993).

${ }^{16}$ A. Buzdin and D. Feinberg, Physica C 256, 303 (1996). 